\title{
Comparación de la bioaccesibilidad de calcio en leche de vaca, semillas de ajonjolí (Sesamun indicum) y almendra (Prunus amygdalus)
}

Comparison of calcium bioaccessibility in cow's milk, sesame seeds (Sesamun indicum) and almond (Prunus amygdalus)

\author{
Narda Lucía Damián Bastidas ${ }^{1, a}$, Elydia Cornelia Mujica Albán ${ }^{2, b}$ \\ ${ }^{1}$ Facultad de Medicina, Universidad Nacional Mayor de San Marcos. Lima, Perú. \\ ${ }^{2}$ Instituto Nacional de Biología Andina, Facultad de Medicina, Universidad Nacional Mayor de San Marcos. Lima, Perú. \\ a Licenciada en nutrición, ORCID: https://orcid.org/0000-0002-0409-6244 \\ ${ }^{\mathrm{b}}$ Doctora en ciencias con mención en fisiología, ORCID: https://orcid.org/0000-0002-8331-9935
}

\section{An Fac med. 2020;81(4):427-31. / DOI: https://doi.org/10.15381/anales.v81i4.18932}

\section{Correspondencia:}

Narda Lucía Damián Bastidas

narda.db@gmail.com

Recibido: 15 de octubre 2020

Aprobado: 21 de noviembre 2020

Publicación en línea: 15 de marzo 2021

Conflictos de interés: Las autoras declaran no tener conflictos de interés.

Fuente de financiamiento:

Autofinanciado

Citar como: Damián N, Mujica E. Comparación de la bioaccesibilidad de calcio en leche de vaca, semillas de ajonjoli (Sesamun indicum) y almendra (Prunus amygdalus). An Fac med. 2020;81(4):427-31.DOI: https://doi. org/10.15381/anales.v81i4.18932

\section{Resumen}

Introducción. La biodisponibilidad de un nutriente refleja la fracción disponible para ser absorbido y utilizado. Resulta critica al evaluar la calidad nutricional de un alimento. Objetivo. Comparar la bioaccesibilidad de calcio entre el ajonjoli (Sesamun indicum) y almendra (Prunus amygdalus) con la bioaccesibilidad de calcio de la leche de vaca utilizando un método in vitro. Métodos. Estudio de tipo observacional que evaluó la bioaccesibilidad mediante el método in vitro de dializabilidad. Resultados. La dializabilidad de calcio de la leche de vaca fue de 20,71\%; en las semillas de ajonjoli y almendra fue $1 \%$ y $2,27 \%$, respectivamente. Se determinó que la leche de vaca cubre entre el $2 \%$ - $4 \%$ del requerimiento total de calcio en niños de 1 a 6 años de edad y del $2 \%$ en adolescentes y adultos. En las semillas, la cobertura fue mínima. Conclusión. La bioaccesibilidad y aporte potencial de calcio de la leche entera de vaca fue superior en comparación con las semillas de almendra y ajonjolí.

Palabras clave: Disponibilidad Biológica; Calcio; Calcio en la Dieta; Leche; Semillas; Sesamum; Prunus (fuente: DeCS BIREME).

\section{Abstract}

Introduction. The bioavailability of a nutrient reflects the fraction available to be absorbed and used. Is critical when evaluating the nutritional quality of a food. Objective. To compare the bioaccessibility of calcium between sesame (Sesamun indicum) and almond (Prunus amygdalus) with the bioaccessibility of calcium from cow's milk using an in vitro method. Methods. An observational study that evaluated bioaccessibility using the in vitro dialyzability method. Results. Calcium dialysability of cow's milk was $20,71 \%$; in sesame and almond seeds it was $1 \%$ and $2,27 \%$, respectively. It was determined that cow's milk covers between $2 \%-4 \%$ of the total calcium requirement in children 1 to 6 years of age and $2 \%$ in adolescents and adults. In seeds the coverage was minimal. Conclusions. The bioaccessibility and potential calcium contribution of whole cow's milk was higher compared to almond and sesame seeds.

Keywords: Biological Availability; Calcium; Calcium, Dietary; Milk; Seeds; Sesamum; Prunus (source: MeSH NLM). 


\section{INTRODUCCIÓN}

La biodisponibilidad se define como la integración de distintos procesos fisiológicos, de bioactividad y bioaccesibilidad, mediante los cuales una fracción de un nutriente ingerido se encuentra disponible para la digestión, absorción, transporte, utilización y eliminación ${ }^{(1,2)}$. Esta variable resulta significativa cuando se evalúa la calidad nutricional de un alimento, especialmente al considerar el contenido de minerales ${ }^{(3)}$. Dentro de los minerales, el calcio es el más abundante y cumple importantes funciones tales como metabolismo óseo, segundo mensajero, transmisión neuromuscular, liberación hormonal y de neurotransmisores, entre otros ${ }^{(4,5)}$. La biodisponibilidad de este mineral se ve afectada por factores intrínsecos y extrínsecos ${ }^{(6)}$, siendo los extrínsecos los que afectan considerablemente la cantidad absorbida, ya que influyen sobre la superficie de absorción de las células intestinales, favoreciendo la absorción como es el caso de los fosfopéptidos de caseína, inulina ${ }^{(7)}$, lactosa y vitamina $\mathrm{D}^{(8)}$ o disminuyéndola como los fitatos, fibra dietética y oxalatos ${ }^{(7)}$.

La fuente principal de calcio son los lácteos y de estos la leche es de amplio consumo a nivel mundial y presenta componentes que favorecen la absorción de calcio en el epitelio intestinal (9,10); algunos investigadores señalan que el efecto de ciertos componentes aún no se encuentra establecido ${ }^{(11)}$. En los últimos años, los sucedáneos de la leche, los cuales tienen como materia prima alimentos de origen vegetal ${ }^{(9)}$, tienen biodisponibilidad que está en discusión; así, en diferentes estudios in vitro se observó una baja biodisponibilidad de calcio debido a la presencia de antinutrientes ${ }^{(12)}$. Sin embargo, otros estudios aseguran que el contenido de minerales y su posterior absorción pueden variar significativamente dependiendo de la fuente vegetal, el país, el clima, las prácticas agrícolas y el procesamiento del alimento ${ }^{(3)}$.

En nuestro país no se han realizado estudios sobre biodisponibilidad en alimentos considerados fuentes importantes de calcio. Conocer la cantidad que es sus- ceptible a ser absorbida de este mineral en los alimentos, es de suma importancia para establecer recomendaciones adecuadas en los diferentes grupos etarios. Por ello en la presente investigación tuvo como objetivo comparar la bioaccesibilidad de calcio del ajonjolí (Sesamun indicum) y almendra (Prunus amygdalus) con la bioaccesibilidad de calcio de la leche de vaca utilizando un método in vitro.

\section{MÉTODOS}

\section{Diseño y lugar de estudio}

Estudio de tipo observacional. La bioaccesibilidad fue determinada mediante la técnica in vitro de dializabilidad desarrollada por Miller y colaboradores ${ }^{(13)}$ y modificado por Wolfgor y colaboradores ${ }^{(14)}$. El procedimiento se realizó en el laboratorio de Farmacia y Bioquímica de la Universidad de Buenos Aires, Argentina.

\section{Reactivos químicos utilizados}

Se utilizó pepsina 1:10000 (Sigma- Aldrich P-7000), extracto biliar (Sigma B-8631), pancreatina (Sigma-Aldrich P-1750), PIPES [piperazine-NN'-bis (2-ethane-sulfonic acid)] disodium salt 99\% (Sigma P-3768), membrana de diálisis Spectra/Por ${ }^{\circledR}$ de 6-8 000 KDa (Spectrum, CA, USA), ácido perclórico 70-72\% (Merck 100519), y ácido nítrico 65\% (Merck 101518).

\section{Materias primas}

Leche entera de vaca marca Laive, semillas de ajonjolí blanco (Sesamun indicum) de la distribuidora Natural Seed y semillas de almendra cruda (Prunus amygdalus) de la distribuidora Frutos y Procesos SAC.

\section{Procesamiento de las materias primas}

Las semillas de ajonjolí y almendra fueron molidas utilizando un molinillo eléctrico Decalab 5000 Gvolt 220 y reservadas en frascos esterilizados en un ambiente seco. La leche de vaca fue separada en tres partes de $200 \mathrm{~mL}$ en beakers de vidrio cubiertos con parafilm y conservadas en refrigeración $\left(T^{\circ} 4\right.$ a $\left.6{ }^{\circ} \mathrm{C}\right)$. Tanto las semillas molidas como la leche de vaca fueron almacenadas por un tiempo aproximado de 24 horas.

\section{Método de dializabilidad}

Se pesaron 20 gramos de las muestras sólidas y 50 gramos de leche en matraces de Erlenmeyer; en el caso de las muestras sólidas se enrasaron con $30 \mathrm{~mL}$ de agua desionizada. Posteriormente se adicionó $5 \mathrm{~mL}$ de alfa-amilasa a cada una de ellas (se consideró una relación de 2,4 g de enzima/30 $\mathrm{mL}$ ), se cubrieron con parafilm y se incubaron por 30 minutos en baño maría $\left(37^{\circ} \mathrm{C}\right)$ con agitación constante $(60 \mathrm{rpm})$. A continuación se midió el $\mathrm{pH}$ y se tituló con $\mathrm{HCl} 6 \mathrm{~N}$ para lograr un $\mathrm{pH}$ de 2, posteriormente se adicionó 1,6 $\mathrm{mL}$ de pepsina a cada Erlenmeyer y se incubó en baño maría $\left(37^{\circ} \mathrm{C}\right)$ con agitación constante por 2 horas; ello para simular el proceso de digestión gástrica. En el caso de la pepsina la relación fue de 16 $\mathrm{g} / 100 \mathrm{~mL}$ y se enrasó con $\mathrm{HCl}$ 0,1 N.

Se traspasó 15 gramos de cada muestra a frascos esterilizados y rotulados, y se colocaron las bolsas de diálisis que contenían 18,75 mL de buffer PIPES Ilevándolas a incubación por una hora. La molaridad y el $\mathrm{pH}$ del buffer se establecieron para cada muestra luego de determinar los mEq totales de cada una y se consideró la relación de $1 \mathrm{M}$ PIPES equivalente a 346,3 $\mathrm{g}$; para dicho procedimiento se utilizó como base las especificaciones establecidas por Drago y colaboradores ${ }^{(15)}$.

Después de la hora de incubación se midió el pH y se colocó $3,75 \mathrm{~mL}$ de la solución de bilis- pancreatina y se llevó a incubar por dos horas en baño maría $\left(37^{\circ} \mathrm{C}\right)$ con agitación constante; la solución de bilis-pancreatina se preparó previamente y para ello se consideró la relación de 2,5 $\mathrm{g}$ de bilis en $100 \mathrm{~mL}$ y 0,4 g de pancreatina en $100 \mathrm{~mL}$ las que fueron enrasadas con $\mathrm{NaHCO}_{3}$ 0,1 N.

Transcurridas las dos horas se removieron las bolsas de diálisis, se enjuagaron con agua desionizada y se pesaron los contenidos en tubos de mineralización.

Para la lectura de los minerales se siguió el procedimiento de espectrofotometría de absorción atómica (16) y se utilizó una mineralización por vía húmeda. Para ello, al contenido de las bolsas de diálisis después de ser pesadas se les colocó $5 \mathrm{~mL}$ de ácido nítrico y $5 \mathrm{~mL}$ de ácido perclórico y los tubos de minerali- 
zación fueron llevados al mineralizador donde estuvieron un aproximado de 12 horas. El contenido se traspasó a tubos de ensayo y se enrasó a $20 \mathrm{~mL}$ con agua desionizada y antes de la lectura en el espectrofotómetro se diluyó con cloruro de lantano para evitar la interferencia de los fosfatos; para el ajonjolí se hizo una dilución de 1:50 y para la leche y la almendra fue de 1:10.

Para el cálculo de la dializabilidad se utilizó la siguiente fórmula:

Dializabilidad \% del mineral $=\frac{\mathrm{mg} \text { de mineral dializado }}{\mathrm{mg} \text { de mineral dializado }} \times 100$

Se calculó el aporte potencial de calcio en las muestras considerando la siguiente fórmula:

$\left[\mathrm{Ca}^{+2}\right]$ en $100 \mathrm{~g}$ de muestra $\times$ \% 100

\section{Análisis estadístico}

Se utilizó el programa estadístico STATA versión 14. Las variables numéricas fueron resumidas mediante promedio y desviación estándar. Los aportes potenciales y porcentajes de cobertura fueron determinados en base a $100 \mathrm{~mL}$ para la leche o 100 g para las semillas y expresado con medida de tendencia central y de dispersión.

\section{RESULTADOS}

La semilla de ajonjolí en 100 g presentó valores de aproximadamente 1 gramo de calcio y cuando se analizó el porcentaje dializado se obtuvo un porcentaje de $1 \%$. En el caso de la leche el porcentaje de dializabilidad fue de $20 \%$ y en la almendra de $2 \%$ (Tabla 1 ).

En relación al aporte potencial, que representa la cantidad potencialmente absorbible de calcio, se obtuvo que en el grupo etario de 1 a 6 años la leche de vaca cubre el $4 \%$ del requerimiento diario de calcio en niños de 1 a 3 años y del $3 \%$ en niños de 4 a 6 años, mientras que la almendra cubre el $0,6 \%$ y $1 \%$ respectivamente; en el caso del ajonjoli el porcentaje de cobertura fue de 1 a $2 \%$, respectivamente, en cada grupo poblacional (Tabla 2).

En el caso de los adolescentes y adultos la leche de vaca cubre el 1,6\% del requerimiento para adolescentes y de $2,1 \%$ para adultos. La almendra cubre el 0,4\% en adolescentes y el 0,5\% en adultos; el ajonjolí cubre alrededor del $1 \%$ del requerimiento diario en este grupo poblacional (Tabla 3).

\section{DISCUSIÓN}

El porcentaje de dializabilidad de la leche de vaca fue de $20,71 \%$, valor que es similar al reportado por Roig y col. que obtuvieron un porcentaje de dializabilidad de $20 \pm 0,9 \%{ }^{(17)}$, la diferencia se debería a la membrana de diálisis utilizada, que fue de 10000 - 12000 Da en el estudio mencionado. Soto y col. determinaron que la dializabilidad de calcio para la leche fue de 23,19 \pm 0,02 \%, valor superior al obtenido en nuestra investigación, lo que se explicaría por la diferencia metodológica, ya que el estudio de Soto utilizó el método de Caco-2 que emplea una monocapa de células con función de absorción ${ }^{(18)}$; en dicho estudio no se especificó el tipo de leche utilizada o si la misma fue suplementada con calcio.

En el caso de la almendra molida se obtuvo un valor aproximado de $2 \%$, el cual resulta inferior a los estudios realizados por Weaver y Kafaoglu y col. que obtuvieron valores

Tabla 1. Valores de calcio y porcentaje de calcio dializado de acuerdo al tipo de muestra estudiada.

\begin{tabular}{lccccc} 
& \multicolumn{2}{c}{ Calcio $^{\text {a,b }}$} & & \multicolumn{2}{c}{ \% calcio dializado $^{c}$} \\
\cline { 2 - 3 } \cline { 6 - 6 } & Promedio & DE & & Promedio & DE \\
\hline Leche de vaca & 102,94 & 8,29 & & 20,71 & 3,62 \\
\hline Almendra molida & 229,61 & 10,71 & & 2,71 & 0,33 \\
\hline Ajonjolí molido & 967,70 & 51,96 & & 1,01 & 0,18 \\
\hline
\end{tabular}

DE: desviación estandar

${ }^{a}$ Se analizaron 4 muestras en el caso de la leche de vaca y 3 muestras en el caso de la almendra y el ajonjolí debido a contaminación.

${ }^{b}$ Valores expresados en $\mathrm{mg}$ por $100 \mathrm{ml}$ de la muestra analizada para el caso de la leche y en $\mathrm{mg} / 100 \mathrm{~g}$ para almendra y ajonjolí.

${ }^{c}$ El porcentaje de calcio dializado fue determinado en base a 4 muestras en el caso de la almendra, 3 en la leche de vaca y en el ajonjoli debido a contaminación. de $21,2 \%{ }^{(19)}$ y $6,9 \%{ }^{(20)}$, respectivamente. La diferencia de resultados puede explicarse por el método utilizado, que en el caso de Weaver fue un método in vivo realizado en ratas ${ }^{(19)}$ y en el de Kafaoglu se utilizó el método PBET que utiliza componentes químicos diferentes ${ }^{(20)}$. Considerando la dializabilidad de la semilla de ajonjolí se obtuvo un valor de $1 \%$, inferior al obtenido en un estudio realizado en Tailandia donde se reportó un este estudio el procesamiento de la semilla fue diferente lo que pudo conducir a la variación de resultados. Weaver en su estudio obtuvo un valor de dializabilidad de $20,8 \%$, valor muy superior al de la presente investigación, que pudo deberse al uso de una metodología in vivo ${ }^{(19)}$ y los resultados pueden variar dependiendo de la especie, actividad enzimática y estado fisiológico ${ }^{(8,22)}$.

Tanto en la almendra como en el ajonjolíllama la atención la dializabilidad de calcio, considerando que ambas semillas tienen una cantidad de calcio de $230 \mathrm{mg} / 100 \mathrm{~g}$ y $968 \mathrm{mg} / 100 \mathrm{~g}$, respectivamente, lo que llevaría a considerarlas como fuentes importantes de calcio. Sin embargo, se debe considerar la presencia de antinutrientes como un limitante importante de absorción de calcio y el ácido oxálico es uno de ellos, ya que forma complejos insolubles con este mineral ${ }^{(23,24)}$, y la semilla de ajonjolí posee más de 500 mg/100g de ácido oxálico lo que se correlaciona de forma negativa con la biodisponibilidad de calcio de este alimento ${ }^{(25)}$. Por otro lado, los fitatos a $\mathrm{pH}$ fisiológico presentan carga negativa y se unen a cationes divalentes ${ }^{(25)}$ formando porcentaje de $4,2 \%{ }^{(21)}$, cabe resaltar que en 
Tabla 2. Aporte potencial de calcio en $100 \mathrm{ml} \mathrm{o} 100 \mathrm{~g}$ de muestra y porcentaje de cobertura del requerimiento diario en niños de 1-6 años

\begin{tabular}{lccc} 
& Aporte potencial & $\begin{array}{c}\text { \% cobertura para 1 a } \\
\text { 3 años }\end{array}$ & $\begin{array}{c}\text { \% cobertura para } \mathbf{4} \\
\text { a } \mathbf{6}^{\text {años }}{ }^{\text {b }}\end{array}$ \\
\hline Leche de vaca $\bar{x}(+D E)$ & $20,5( \pm 2,36)$ & 4,1 & 2,6 \\
\hline Almendra molida $\bar{x}(+D E)$ & $5,2( \pm 2,77)$ & 1 & 0,6 \\
\hline Ajonjolí molido $\bar{x}(+D E)$ & $10,9( \pm 0,91)$ & 2,2 & 1,4
\end{tabular}

a Basado en el requerimiento diario de calcio recomendado por la OMS para 1 a 3 años (500 mg/día)

Basado en el requerimiento diario de calcio recomendado por la OMS para 4 a 6 años (800 mg/día)

Tabla 3. Aporte potencial de calcio en $100 \mathrm{ml}$ o $100 \mathrm{~g}$ de muestra y porcentaje de cobertura del requerimiento diario en los diferentes grupos estarios

\begin{tabular}{lccc} 
& Aporte potencial & $\begin{array}{c}\text { \% cobertura para 10 a } \\
\mathbf{1 8} \text { años }^{\mathbf{c}}\end{array}$ & $\begin{array}{c}\text { \% cobertura para } \\
\text { 19 a más años }\end{array}$ \\
\hline Leche de vaca $\bar{x}(+D E)$ & $20,5( \pm 2,36)$ & 1,6 & 2,1 \\
\hline Almendra molida $\bar{x}(+D E)$ & $5,2( \pm 2,77)$ & 0,4 & 0,5 \\
\hline Ajonjolí molido $\bar{x}(+D E)$ & $10,9( \pm 0,91)$ & 0,8 & 1,1 \\
\hline
\end{tabular}

Basado en el requerimiento diario de calcio recomendado por la OMS para 10 a 18 años (1300 mg/día)

dBasado en el requerimiento diario de calcio recomendado por la OMS para 19 a más años (1000 mg/día)

complejos insolubles en el intestino impidiendo la absorción de calcio y favoreciendo su excreción por las heces ${ }^{(26,27)}$. Schlemmer y colaboradores determinaron que las almendras contienen 0,35 - 9,42g/100g y en el caso del ajonjolí el valor oscila entre $1,44-5,36 \mathrm{~g} / 100 \mathrm{~g}{ }^{(28)}$.

Entre las limitaciones del estudio se puede mencionar el uso de una técnica in vitro frente a una técnica in vivo de biodisponibilidad. No obstante, la técnica de dializabilidad es ampliamente utilizada para conocer como la matriz alimentaria influye en el proceso de absorción mineral y su aceptación es universal comparada con las técnicas en vivo que al utilizar radioisótopos puede ser riesgosa y en el caso de los animales hay variabilidad dependiendo de la especie y comportamiento enzimático y estado fisiológico.

Considerando la información anterior se puede afirmar que las semillas de ajonjolí y almendra presentan resultados controversiales en relación a su dializabilidad; por un lado en nuestro estudio y en la de otros autores poseen un valor de dializabilidad bajo comparado con la leche de vaca, mientras que en el estudio de Weaver la dializabilidad es similar al de la leche; sin embargo, en este último caso se debe considerar que el método utilizado fue diferente al nuestro y ello pudo repercutir en los valores obtenidos.

Se concluye que la leche de vaca posee una bioaccesibilidad mayor de calcio a diferencia de las semillas de almendra y ajonjolí cuya bioaccesibilidad es baja.

\section{AGRADECIMIENTO}

A la Dra. Maria Julieta Binaghi, jefa de trabajos prácticos de la Cátedra de Bromatología de la Facultad de Farmacia y Bioquímica de la Universidad de Buenos Aires por su asesoría en el desarrollo de la parte experimental de la presente investigación.

\section{REFERENCIAS BIBLIOGRÁFICAS}

1. Haro-Vicente J, Martínez-Graciá G. Optimisation of in vitro measurement of available iron from different fortificants in citric fruit juices. Food Chem. 2006; 98: 638-48. DOI: https://doi.org/10.1016/j. foodchem.2005.06.040

2. Klobukowski J, Skibniewska K, Kowalski I. Calcium bioavailability from dairy products and its release from food by in vitro digestión. J. Elem. 2014;19(1):277-88. DOI: 10.5601/jelem.2014.19.1.436

3. Suliburska J, Krejpcio Z. Evaluation of the content and bioaccessibility of iron, zinc, calcium and magnesium from groats, rice, leguminous grains and nuts. J Food Sci Technol. 2014;51(3):589-94. DOI: 10.1007/s13197-011-0535-5

4. Farré R. La leche y los productos lácteos: fuentes dietéticas de calcio. Nutr Hosp. 2015;31(2):1-9. DOI: 10.3305/nh.2015.31.sup2.8676

5. Gallagher, M. Los nutrientes y su metabolismo. En: Mahan K, Escott-Stum S, autores. Terapia de alimentación y nutrición de Krause: Elsevier Masson; 2008:103-7.

6. Binaghi M, Pellgrino N, Valencia M. Bioaccesibilidad de minerales en infusiones de yerba mate (llex paraguariensis St) y en mezclas con leches fortificadas con hierro. ALAN. 2011;61(1):81-6.

7. Etcheverry P, Grusak M, Fleige L. Application of in vitro bioaccessibility and bioavailability methods for calcium, carotenoids, folate, iron, magnesium, polyphenols, zinc and vitaminas B6, B12, D and E. Front Physiol. 2012;(3):1-22. DOI: 10.3389/ fphys.2012.00317

8. Caldera Y. Biodisponibilidad in vitro de hierro $y$ calcio en cereales y derivados. (Tesis de Maestria). Valencia-España: Universidad de Valencia; 2012;137pp.

9. Scholz-Ahrens K, Ahrens F, Barth C. Nutritional and health attribute of milk and milk imitations. Eur J Nutr. 2019;59(1):19-34. DOI: 10.1007/s00394019-01936-3

10. Perales S, Barberá R, Lagarda M, Farré R. Bioavailability of calcium from milk-based formulas and fruit juices containing milk and cereals estimated by in vitro methods (solubility, dializability, and uptake and transport by caco-2 cells. J Agric Food Chem. 2005;(53):3721-6. DOI: 10.1021/jf047977y

11. Sánchez L, Hernández M, López F. Regulación y biodisponibilidad efectiva del calcio. Influencia de la Vitamina D. En: Martínez, J, De Arpe, C, editores. Avances en Alimentación, Nutrición y Dietética. Sociedad Española de Dietética y Ciencias de la Alimentación; 2012:51-9.

12. Weaver M, Proulx W, Heaney R. Choices for achieving adequate dietary calcium with a vegetarian diet. Am J Clin Nutr. 1999;70(3):543S-48S. DOI: 10.1093/ajcn/70.3.543s

13. Miller D, Schricker B, Rasmussen R, Campen, D. $A n$ in vitro method for estimation of iron availability from meals. Am J Clin Nutr. 1981;34(10): 2248-56. DOI: 10.1093/ajcn/34.10.2248

14. Wolfgor R, Drago S, Rodriguez V, Pellegrino N, Valencia M. In vitro measurement of available iron in fortified foods. Food Research International. 2002;35(1):85-90. DOI: 10.1016/S09639969(01)00122-3

15. Drago S, Binaghi M, Valencia M. Effect of gastric digestion $\mathrm{pH}$ on iron, zinc, and calcium dializability from preterm and term starting infant formulas. Journal of Food Science. 2005;70(2):S107-12. DOI: 10.1111/j.1365-2621.2005.tb07113.x

16. Association of Official Analytical Chemists. Official Method of Analysis of AOAC International (17 ed.). Washington-USA; 2000.

17. Roig M, Alegría A, Barberá R, Farré R, Lagarda M. Calcium dialysability as an estimaton of bioavailability in human milk, cow milk and infant formulas. Food Chemistry. 1999;64(3):403-9. DOI: https://doi. org/10.1016/S0308-8146(98)00151-4

18. Soto A, Morales P, Haza A, Garcia M, Selgas M. Bioavailability of calcium from enriched meat products using Caco-2 cells. Food Research International. 2014;(55):263-70. DOI: 10.1016/j. foodres.2013.10.038 
19. Weaver C, Plawecki K. Dietary calcium: adequacy of a vegetarian diet. Am J Clin Nutr. 1994;59(5):1238S41S. DOI: 10.1093/ajcn/59.5.1238S

20. Kafaoglu B, Fisher A, Hill S, Kara D. Determination and evaluation of element bioaccessibility in some nuts and seeds by in-vitro gastro-intestinal method. Journal of Food Composition and Analysis. 2014;45:58-65. DOI: 10.1016/j.jfca.2015.09.011

21. Kamchan A, Puwastein P, Sirichakwal P, Kongkachuichai R. In vitro calcium bioavailability of vegetables, legumes and seeds. Journal of Food Composition and Analysis. 2004;17(34):311-20. DOI: 10.1016/j.jfca.2004.03.002

22. Farré R. Biodisponibilidad mineral: evaluación. ANS. 2010;17(1):1-8.
23. Gibson R, Perlas L, Hotz C. Improving the bioavailability of nutrients in plant foods at the household level. Proc Nutr Soc. 2006;65(2):160- 8. DOI: 10.1079/pns2006489

24. Weaver C, Heaney R, Nickel K, Packard P. Calcium bioavailability from high oxalate vegetables: Chinese vegetables, sweet potatoes and rhubarb. Journal of Food Science. 1997;62(3):524-5. DOI: 10.1111/J.1365-2621.1997.TB04421.X

25. Embaby $H$. Effect of heat treatments on certain antinutrientes and in vitro protein digestibility of peanut and sesame seeds. Food Science and Technology Research. 2010;17(1):31-8. DOI: https:// doi.org/10.3136/fstr.17.31
26. Al Hasan S, Hassan M, Saha S, Islam M, Billah M, Islam S. Dietary phytate intake inhibits the bioavailability of iron and calcium in the diets of pregnant women in rural Bangladesh: a cross-sectional study. BMC Nutr. 2016;2(1):1-10. DOI: 10.1186/s40795-016-0064-8

27. Gibson R. The role of diet- and host-related factors in nutrient bioavailability and thus in nutrient-based dietary requirement estimates. Food Nutr Bull. 2007;28(1):S77-S100. DOI: 10.1177/15648265070281S108

28. Schlemmer U, Frolich W, Prieto R, Grases F. Phytate in foods and significance for humans: foods sources, intake, processing, bioavailability, protective role and analysis. Mol Nutr Food Res. 2009; 53 (2):S330-75. DOI: 10.1002/mnfr.200900099 